\title{
Relación entre habilidades sociales y acoso escolar ${ }^{1}$
}

\section{Relation between social skills and bullying}

DOI: http://dx.doi.org/10.17981/cultedusoc.8.2.2017.05

Dolly Enith Vargas Martínez ${ }^{2}$ y Yuri Susana Paternina ${ }^{3}$

\section{Resumen}

El acoso escolar es un fenómeno que se presenta frecuentemente en las relaciones entre estudiantes, que asumen diversos roles y que quizás encuentran esta manifestación como una forma de interactuar con los otros. Numerosos estudios se han hecho para evaluar esta problemática y se han propuesto diversas intervenciones, sin embargo, se observa que el fenómeno sigue presentándose. En este trabajo se pretende mostrar la importancia de las habilidades sociales en el afrontamiento del acoso escolar. La investigación ha sido desarrollada bajo la metodología descriptiva, a través de la selección de artículos en las bases de datos Redalyc, Scielo, Revista Investigación Psicoeducativa, Behavioral Psychology, Revista Iberoamericana de Psicología: Ciencia y Tecnología, Liberabit Revista de Psicología, Revista psicología, Cultura y Sociedad, se utilizaron descriptores como: skills and bullying, acoso escolar, habilidades sociales, en los últimos 15 años.

Palabras clave: Acoso escolar, habilidades sociales.

Recibido: Agosto 10 de 2017 / Aceptado: Octubre 20 de 2017.

\footnotetext{
${ }^{1}$ El presente artículo es el resultado de la investigación del grupo de investigación Psicología integral y Desarrollo humano de la Facultad de Psicología de la Fundación Universitaria los Libertadores, Colombia en convenio con la Universidad de Granada, España, titulado "Desarrollo de un programa de entrenamiento en habilidades sociales con formato lúdico para la prevención e intervención del acoso escolar en niños y niñas de 10 a 15 años” con código PSI-008-17, inscrito a la línea de investigación Desarrollo Humano Integrador en el Contexto Social Colombiano, que comenzó en febrero de 2017 y finalizó en Diciembre de 2017.

${ }^{2}$ Magíster en Desarrollo educativo y social. Investigadora principal del proyecto de investigación. Docente tiempo completo de la Facultad de Psicología de la Fundación Universitaria Los Libertadores, e-mail: devargasm@libertadores.edu.co

${ }^{3}$ Psicóloga Fundación Universitaria Los Libertadores. Pasante investigativa de la Facultad de Psicología de la Fundación Universitaria Los Libertadores, e- mail: yspaterninar@libertadores.edu.co
}

The author; licensee Universidad de la Costa - CUC. Cultura, Educación y Sociedad vol. 8 no. 2, pp. 61-78. Julio - Diciembre, 2017 Barranquilla. ISSN 2145-9258 Impreso, ISSN 2389-7724 Online 


\begin{abstract}
Bullying is a phenomenon that frequently occurs in relationships between students, who assume diverse roles and who may find this manifestation as a way of interacting with others. Numerous studies have been done to evaluate this problem and several interventions have been proposed, however, it is observed that the phenomenon is still present. This paper aims to show the importance of social skills in coping with bullying. The research has been developed under the descriptive methodology, through the selection of articles in the databases Redalyc, Scielo, Revista Psicoeducativa, Behavioral Psychology, Revista Iberoamericana de Psicología: Ciencia y Tecnología, Liberabit Revista de Psicología, Revista psicología, Culture and Society, using descriptors such as: skills and bullying, bullying, social skills, in the last 15 years.
\end{abstract}

Keywords: Bullying, social skills.

\title{
Introducción
}

El presente artículo presenta el resultado de la revisión teórica de la relación entre las habilidades sociales y el acoso escolar en niños y niñas, con el fin de brindar información actualizada que ayude a la comprensión teórica de la relación entre estos dos constructos. .

De acuerdo con Gairín, Armengol y Silva (2013), "el fenómeno del acoso escolar ha existido siempre y se ha dado en todo tipo de centros educativos: privados o públicos, selectivos o marginales, situados en grandes ciudades o en pueblos pequeños" (p. 24). $\mathrm{El}$ acoso escolar es una forma de agresión, perjudicial e intencionada (Trianez, Muñoz \& Jiménez, 2007; citado por Polo, Barco, Castaño, Bullon, \& Carroza, (2015) que afecta el bienestar social, emocional y psicológico de la persona que lo sufre (Estévez, Murgui y Musitu, 2008). También, es un comportamiento en el cual los ataques hacia la víctima se vuelven sistemáticos, donde se encuentra una situación de persecución psicológica y/o física (Olweus, 1983; citado por Polo et al., 2015). Autores como Uribe, Orcasita y Gómez (2012) refieren que para comprender el fenómeno del acoso escolar es fundamental conocer dos características que lo identifican. La primera de ellas es la presencia intrínseca de una relación de poder "dominancia-sumisión" que poseen los victimarios sobre otra persona que es la víctima. La segunda es que, en el acoso escolar, las situaciones de agresión se dan de forma frecuente.

En este sentido, el bienestar psicológico, emocional y social son factores que se ven afectados en víctimas de acoso escolar y si no se trata profesionalmente, a tiempo y bajo la rigurosidad de las leyes, puede tener consecuencias de salud, baja autoestima, suicidio, tristeza, ausentismo, deficiencia en las relaciones interpersonales y en la comunicación efectiva. De éste modo como lo dice la Ley 1098 del 2006, en su artículo 18, (Procuraduría General de la Nación, 2010):

"Los niños, las niñas y los adolescentes tienen derecho a ser protegidos contra todas las acciones o conductas que causen muerte, daño o sufrimiento físico, sexual o psicológico. Tienen derecho a la protección contra el maltrato y los abusos de toda índole por parte de sus padres, de sus representantes legales, de las personas responsables de su cuidado y de los miembros de su grupo familiar, escolar y comunitario". (p. 25) 
Así las cosas, para cualquier persona que atente contra la integridad de un niño o niña, en el contexto escolar, las consecuencias legales pueden ser de índole agravante, ya que todos los seres humanos tenemos derecho a convivir en un ambiente sano, libre de humillación, burla, crítica, trato negligente entre otros posibles. De este modo, la percepción de esta problemática en la actualidad, corresponde a la forma en que las familias y los medios de comunicación la están haciendo visible (Gairín, et al., 2013). De igual forma, el acoso escolar presenta innumerables comportamientos que son manifestados dentro del contexto escolar como ataques, amenazas, insultos, burlas, agresiones, exclusión social, rechazo y daño a los objetos personales (Olweus, 1998; citado por Gómez, 2015).

Por su parte, Piñuel y Oñate (2005; citados por Uribe, Orcasita \& Gómez, 2012) definen el acoso escolar como un prolongado e intencionado maltrato verbal que recibe un niño, niña o adolescente por parte de uno o varios compañeros que presentan comportamientos crueles con el fin de asustarlo, someterlo, amenazarlo, atentando contra su dignidad. Más sin embargo, Marín \& Reidl (2013) lo definen como el propósito de causar daño, pero resaltan que en los vínculos de amistad entre los alumnos se manifiestan conductas que posiblemente pueden estimarse como agresivas pero no precisamente se ejecutan con la intención de causar daño; más bien, son modos que se han establecido con consentimiento, implícito o explícito, de no producir malestar, incomodidad o sufrimiento y de acuerdo con Ortega et. al., (2001; referido por Enríquez y Garzón, 2015) las agresiones que son consideradas como acoso escolar son las que no tienen justificación, son hirientes y perjudiciales desencadenando perjuicios psicológicos en la víctima, siendo de esta manera los agresores conscientes del sufrimiento que provocan en la otra persona hasta llegar al punto de someterlo en condición de estrés. De éste modo, Alcantará (2008; citado en Enríquez y Garzón, 2015), insiste en la concepción de acoso escolar como una intimidación y maltrato entre escolares, de forma sistemática y con el deseo de humillar a una persona indefensa, a través de agresiones físicas, verbales y psicológicas hacia la víctima. Desde la perspectiva social se define al acoso escolar como el escenario de violencia mantenida, mental o física de un alumno o varios contra otro sujeto del grupo que no posee la suficiente capacidad de defenderse (Sánchez \& Cerezo 2011).

En este orden de ideas, en Colombia la Ley 1620 del 2013 en su artículo II, refiere que el acoso escolar es una (Congreso de Colombia, 2013):

"Conducta negativa, intencional metódica y sistemática de agresión, intimidación, humillación, ridiculización, difamación, coacción, aislamiento deliberado, amenaza o incitación a la violencia o cualquier forma de maltrato psicológico, verbal, físico o por medios electrónicos contra un niño, niña o adolescente, por parte de un estudiante o varios de sus pares con quienes mantiene una relación de poder asimétrica, que se presenta de forma reiterada o a lo largo de un tiempo determinado. También puede ocurrir por parte de docentes contra estudiantes, o por parte de estudiantes contra docentes, ante la indiferencia o complicidad de su entorno. El acoso escolar tiene consecuencias sobre la salud, el bienestar emocional y el rendimiento escolar de los estudiantes y sobre el ambiente de aprendizaje y el clima escolar del establecimiento educativo". $(\mathrm{p}, 2)$ 
Es conveniente mencionar que la violencia escolar causa en la mayoría de los casos, vulnerabilidad relacional, que es la base para que se presente conflicto, violencia, ausentismo y sin duda alguna, un menor rendimiento académico (Cornelass, 2010; citado en Stan \& Galea, 2014). Además de esto, se hace alusión a que el foco actual de los investigadores de violencia escolar son eventos de tipo intimidante. El fenómeno del acoso escolar, es una forma precisa de violencia escolar, que se presenta actualmente en todo el mundo y cambia de un individuo a otro en diferentes formas e intensidades. (Krug, Dahlberg, Mercy, Zwi \& Rafael, (2002), Currie, Gabhainn, Godeau, Roberts, Smith, Picket, Richter, Morgan, \& Barnekow, (2008)).

Ahora bien, estudios realizados en España como los de Ayerbe, et. al, (2005) y los de Sánchez, ( 2007) citados por Gairín et al., (2013) muestran que uno de cada cinco estudiantes se halla involucrado en éste tipo de procesos como víctima, como agresor o como ambos actores a la vez. Estos estudios estiman que es un tipo de fenómeno que se encuentra vinculado a situaciones como ambientes familiares específicos o fracaso escolar, aplicable a cualquier clase social; indicando además de esto, que los sujetos agentes del acoso escolar presentan una ausencia de interiorización de las normas.

\section{Tipos de acoso escolar}

Existen diferentes tipos de acoso escolar, que de acuerdo con Toledo \& Magendzo, (2011), son:

1. Físico: que se refiere a golpes, empujones, puños, patadas, halar el pelo vandalismo, esconder o hacer daño a los objetos personales.

2. Verbal: caracterizada por el empleo de palabras con contenido humillante, grosero, descalificador, burlesco, amenazante, irónico, burlón, etc.

3. Relacional o psicológico: corresponde a la exclusión social, aislamiento, indiferencia, rechazo a otro u otros por parte de un individuo o de un grupo.

4. Virtual o cyberbullying: cuando se agrede permanente a alguien a través de los medios electrónicos y de las redes sociales.

En este sentido, es importante hablar de los actores del acoso escolar, lo que permite quizás tener una mejor comprensión del fenómeno, entre los que se encuentran el agresor, la víctima, el observador, los docentes, entre otros. Cerezo (2002) manifiesta que el agresor o victimario presenta un perfil específico frente a la víctima, mostrando provocación, fortaleza física y carácter de liderazgo frente a la ansiedad, cobardía y sentimientos de antipatía que caracterizan a las víctimas. Según Olweus (1993; citado en Polo et. al, 2015) el acosador presenta un estilo de socialización preciso, tiene dificultad para ponerse en el lugar de los otros, muestra deficiencia en autocontrol de las relaciones sociales y con el tiempo se acostumbra a vivir abusando de los demás lo que le genera un impedimento para incorporarse de forma adecuada a la vida social dentro del contexto escolar (Benítez \& Justicia, 2006). Así mismo, al acosador en general se le señala con temperamento agresivo e impulsivo, falta 
de empatía hacia el sufrimiento de su compañero victimizado y ausencia de sentimiento de culpa, son vehementes y necesitan autoritariamente dominar a los demás (Castro, 2011). Por su parte, se encuentra la víctima quien recibe las agresiones de otro de manera sistemática, divulgando la percepción hostil al conjunto del ambiente escolar y desarrollando, entre otros estados de ansiedad y depresión (León, 2009 y Perren \& Alsaker, 2006, citado por Polo, et. al, 2015) así, como disminución de la autoestima y confianza en sí mismo, problemas para adaptarse socialmente y en situaciones extremas, incluso llegando al suicidio (Martínez, -Monteagudo, Inglés, Trianes, García-Fernández, 2011). Por último, se encuentran los observadores que para Musri, (2012), no se encuentran libres de consecuencias, ya que sienten temor de ser acosados y adquieren también modos inadecuados de abordar las relaciones con los demás. Los observadores por un lado, desarrollan el aprendizaje que mediante conductas agresivas se obtiene poder en las relaciones interpersonales y terminan considerando la violencia con los demás como algo inevitable, adaptándose a vivir en un clima de tensión en el que las personas fuertes tienen poder sobre las débiles. Además, aprenden a no ayudar al débil, a no involucrarse y a pasar por alto casos de injusticia y abuso. Cowie (1998) refiere como consecuencia la desensibilización que se origina en los observadores ante el sufrimiento de otros a medida que van considerando acciones reiteradas de agresión en la que no tienen la capacidad de intervenir para evitarlas.

Según, Gairín et al., (2013) el papel que juega el docente se reduce a la transmisión de conocimientos con poca intervención fuera de los límites del aula y menciona que:

"Una sociedad que promueve el desarrollo personal y los valores a través de todos los medios a su alcance disminuye la presencia del fenómeno. Por el contrario, sí un país promueve las desigualdades personales y la violencia como método para la resolución de conflictos, hace posible que el bullying se esconda detrás de cada esquina (p. 25).

De éste modo, estudios realizados en ciencias sociales como los de Michelson, Sugai, Wood \& Kazdin, (1987) y Ferreira \& Muñoz, (2011), sostienen que el problema del acoso escolar se presenta por las dificultades y problemas en las relaciones interpersonales y en las habilidades sociales, por ende, se identifica como un fracaso en el aprendizaje de la competencia social, que tiene gran incidencia tanto en el funcionamiento presente como en el futuro desarrollo de los niños, las niñas y los adolescentes, permitiendo que vayan asimilando los roles, las reglas y las normas sociales.

Por otro lado, Collel y Escudé (2002; citados por Uribe et. al, 2012) proponen que las relaciones entre iguales no se dan sin conflictos y que estos por sí mismos no son malos; refieren que no es adecuado cuando se convierten en situaciones de riesgo y se tornan formas de abuso de poder o cuando ésta es la manera habitual de relacionarse, como se manifiesta en el acoso escolar. De ésta forma, las conductas de abuso de poder entre iguales si se ostentan de forma persistente a través de un periodo de tiempo largo, pueden desarrollar trastornos mentales y episodios críticos en las personas en la adolescencia y en la edad adulta. 


\section{Consecuencias del acoso escolar}

El acoso escolar tiene consecuencias sobre la salud, el bienestar emocional, el rendimiento escolar de los alumnos, sobre el ambiente de aprendizaje y el clima escolar de la Institución Educativa (Enríquez y Garzón, 2015). Además, según Collel y Escudé (2002; citados en Uribe et. al, 2012) los niños, niñas y adolescentes victimizados exhiben altas tasas de síntomas psicosomáticos, ansiedad, depresión, baja autoestima, soledad, aislamiento, baja adaptación escolar y baja concentración; en el grupo de las niñas se han observado especialmente, trastornos de la ingesta como anorexia y bulimia. Ahora bien, Petrosino (2010; citado en Enríquez y Garzón, 2015), señala que:

"La persona que padece la agresión, llega a pensar que lo que pasa es por algún motivo preciso provocando su introversión, además la constitución física de la víctima suele ser débil y va acompañada, de algún tipo de desventaja y una sensación de indefensión" (p, 227).

El grupo de agresores tiende a mostrar ansiedad, depresión y trastorno por déficit de atención con hiperactividad y una tendencia a desarrollar una personalidad antisocial en la adultez. De esta manera, se dice que el acoso escolar no solo afecta a quien lo padece, sino a todos los participantes en general debido a que puede producir entre ellos, de acuerdo con Fernández \& Ruiz, (2009; citados en Enríquez y Garzón, 2015):

"El deterioro de la autoestima, ansiedad, fobia escolar, depresión e intentos de suicidio que suscitan de forma negativa en la salud mental de la víctima. Para los que agreden, las conductas de acoso pueden tornarse crónicas convirtiéndose así en la manera en cómo buscan alcanzar sus metas, corriendo el riesgo de que en la adolescencia o adultez puedan desviarse hacia conductas de violencia doméstica, de género o delictivas y los observadores pueden convertirse en insensibles frente a las múltiples agresiones que ocurren en su entorno no reaccionando ante lo sucedido" (p, 228).

Por otra parte, estudios como los de Kokkinos \& Kipritsi (2012), concluyen que la victimización se halla relacionada con falta de regulación emocional, control de impulsos y con niveles bajos de empatía en el componente cognitivo y si la víctima no tiene la capacidad de reconocer, comprender, regular sus emociones y así mismo expresar sus emociones o las de sus compañeros esto posiblemente puede ocasionar exclusión por parte de ellos y además puede ser un fuerte predictor de ser una víctima de acoso escolar. A partir de estos hechos, se producen diversos factores que intervienen en que un estudiante se pueda convertir en víctima como lo es la baja popularidad, no tener buenas relaciones interpersonales con sus compañeros, bajo manejo de sus sentimientos, timidez e inseguridad, poca asertividad y poco manejo de habilidades sociales (Garaigordobil \& Oñederra, 2010). Para, autores como Mavroveli, Petrides, Sangareau \& Furnham (2009) el niño, niña y adolescente victimizado es más empático porque es más sensible a las consecuencias emocionales de las trasgresiones a las normas. Para entender más a fondo lo que significa la empatía Carlo, Mestre, Samper, Tur, \& Armenta, 2010, Carlo, Mestre, McGinley, Samper, Tur, \& Sandman, 2012, citados por Samper, Mestre, Malonda y Mesurado, 2015) refieren que: "es una emoción controlada, orientada a los sentimientos del otro y por tanto contrapuesta a la impulsividad o falta de autocontrol" (p. 850). 
Existen indicadores o señales de observación tanto para docentes como para la familia donde la víctima presenta determinadas conductas, en las cuales se deja entrever que el alumno está siendo objeto de algún tipo de acoso escolar (Musri, 2012). (Ver Tabla 1).

Tabla 1

Señales para la caracterización de posibles víctimas

\begin{tabular}{ll}
\hline \multicolumn{1}{c}{ Señales para la Institución } & \multicolumn{1}{c}{ Señales para la Familia } \\
\hline Quejas somáticas constantemente. & $\begin{array}{l}\text { Miedo de ir al colegio. } \\
\text { Aislamiento social, se muestra ansioso, triste, con } \\
\text { sentimientos de culpabilidad, timidez y con ataques } \\
\text { de rabia inesperados. }\end{array}$ \\
La no participación en salidas pedagógicas. & $\begin{array}{l}\text { Regresan a casa con heridas en el cuerpo } \\
\text { Evidencias físicas. }\end{array}$ \\
$\begin{array}{l}\text { Escritos en puertas de baño y paredes. } \\
\text { Robo de sus objetos personales. }\end{array}$ & Cambio de rutinas y actividades. \\
$\begin{array}{l}\text { Las relaciones con sus pares no son las adecuadas. } \\
\text { Se presentan rumores malintencionados. }\end{array}$ & Aferrarse a la persona que lo cuida. \\
\hline
\end{tabular}

Fuente: elaboración Musri (2012)

\section{Habilidades sociales}

Caballo (2007) plantea que las habilidades sociales se encuentran relacionadas con conductas que le posibilitan a la persona desenvolverse en un contexto intrapersonal o interpersonal generando de esta manera sentimientos, actitudes, deseos, opiniones o derechos de una forma correcta ante alguna situación. Además de esto, las habilidades sociales permiten la resolución de problemas inmediatos así como la disminución de problemáticas futuras.

Para Del Prette y Del Prette (2008) las habilidades sociales orientan al individuo en conductas relacionadas a su competencia social, generando en la persona eficacia en sus relaciones interpersonales. En este sentido, las concepciones de naturaleza multidimensional, especificidad situacional y carácter aprendido, son algunas de las características que presentan los comportamientos en el individuo. Así mismo, Campo, \& Martínez de Biava (2009), Elliot, DiPerna, Mroch \& Lang (2004), García Núñez del Arco, (2005) aluden que durante las últimas décadas, estudios en habilidades sociales han indicado la unión entre competencia social y la salud físicomental al determinar al conjunto de habilidades sociales como factor importante de protección, así como indicador de desarrollo saludable y de calidad de vida. Además, dentro de éstos estudios se muestra que las habilidades sociales inciden tanto en la infancia como en la vida adulta en la autoestima, en la adopción de roles, en la autorregulación del comportamiento y en el rendimiento académico, entre otros posibles (Rodríguez, Rubio \& Expósito, 1995; Kennedy, 1992; Casares, 2002; Bernal, 1998: citados por Lacunza \& Contini, 2011).

En este sentido, se dice que los comportamientos sociales son aprendidos mediante el desarrollo del ciclo vital de la persona, por lo que existen diversas conductas de los niños, niñas y adolescentes que dependen del proceso de socialización para rela- 
cionarse con sus pares, ser amable o reaccionar agresivamente, entre otros posibles. Para Schaffer (1990, citado en Lacunza \& Contini, 2011), las interacciones sociales llevan consigo modelos de comportamientos complejos y sincronizados ejecutados mutuamente por dos o más sujetos.

De igual forma, alude Monjas (2004) que las habilidades sociales son comportamientos aprendidos y no son variables de la personalidad, aunque existen algunas características de la personalidad que posiblemente pueda potenciar el desarrollo de los comportamientos sociales. La capacidad para formar y mantener amistades estables y satisfactorias está determinada por las experiencias de apego y algunos rasgos de personalidad como: la extroversión, la simpatía, la estabilidad y las diversas oportunidades que brinda la escuela, la familia y la comunidad, para conocer a otras personas (Carr, 2007; citado por Lacunza \& Contini, 2011).

Para Martín (2013), la interacción dentro del grupo de pares es fundamental para el desarrollo social y de la personalidad de los niños, niñas y adolescentes, por cuanto es un contexto productivo y positivo en donde se ponen en juego las habilidades sociales y la efectividad de observar y conocer cómo usan de la mejor forma posible los recursos y estrategias. Lamentablemente, de acuerdo con este autor, en el grupo de iguales se manifiestan aspectos negativos de las relaciones, como los conflictos o desacuerdos entre los alumnos, el acoso escolar o la transferencia de conductas disruptivas y de riesgo dentro del grupo.

Según Goldstein, Sprafkin, Gershaw \& Klein (1989), tanto la familia como la escuela son agentes de socialización importantes para los niños, niñas y adolescentes, son transmisoras de conocimientos y guías de valores que se requieren para interactuar en una sociedad. Además, hay que tener en cuenta que en la escuela, se llevan a cabo las primeras relaciones interpersonales fuera del contexto familiar, por lo que se le imputa cada vez con más ahínco responsabilidad en el fomento de la competencia social de los estudiantes para enfrentar situaciones y problemas personales y sociales y se adapten de manera adecuada y satisfactoria a los diversos contextos en los que deseen incorporarse.

Por su parte, Meichenbaum, Butler y Grudson (1981, citados por García, 2010) señalan que la habilidad social debe considerarse dentro de un marco cultural determinado teniendo presente que los patrones de comunicación varían entre culturas y dentro de una misma cultura, dependiendo de factores tales como la edad, la clase social, el sexo y la educación. Wilkinson y Canter, (1982, citados por Lavilla, 2013) mencionan que la conducta considerada correcta en una situación puede ser, inapropiada en otra, trayendo el individuo a la situación, valores, actitudes, creencias, capacidades cognitivas y un estilo único de interacción. Según los autores no puede haber una forma correcta de comportarse que sea universal, sino una serie de enfoques diferentes que pueden variar de acuerdo con el individuo. De esta manera, Arkowitz, 1981; Schroeder y Rakos, 1983; citados por Caballo, (2007) determinan que hay conductas que son evaluadas como no habilidosas, por ejemplo, decir tonterías, agredir físicamente, que pueden ser reforzadas por otras personas. Linehan (1984; citado por García, 2010) refiere que tanto el contenido como las consecuencias deben ser tenidas en cuenta en cualquier tipo de definición de habilidad social, planteando que al constituir el contenido de la conducta socialmente habilidosa y al 
evaluar las consecuencias de esas conductas se puede lograr alguna estimación del grado de habilidad social.

Por otra parte, Clemence (2001) afirma que las habilidades sociales son la capacidad para establecer cogniciones y conductas dirigidas hacia metas interpersonales que sean culturalmente aceptadas, comprenden conductas verbales y no verbales que orientan a la persona a desenvolverse en su relación diaria con los demás. Aprender y desarrollar estas habilidades es primordial para adquirir relaciones satisfactorias en el entorno social, ayudando a solucionar y prevenir problemas interpersonales. Es conveniente mencionar que trabajar en el marco educacional mediante la contribución del mejoramiento en los estudiantes de las habilidades, resulta de vital importancia, debido a que la educación tiene lugar en el contexto social, en el cual la correcta interacción entre estudiantes y docentes, favorece de una forma efectiva el desarrollo educativo, el rendimiento y por ende el proceso social de cada alumno.

\section{Caracteristicas de las habilidades sociales}

Fernández (1994; citado en Lacunza \& Contini, 2011) señala tres características que presentan las habilidades sociales:

1. Heterogeneidad, debido a que incluyen una variedad de comportamientos en diferentes etapas evolutivas, niveles de funcionamientos y en los contextos en los que puede llegar a tener la actividad humana.

2. Naturaleza interactiva, al considerarse una conducta independiente ajustada a los comportamientos de los interlocutores en un contexto determinado

3. Especificidad situacional, por lo que resulta fundamental la consideración de los contextos socioculturales.

Lacunza, Caballero \& Contini (2012) consideran que la conducta social de los adolescentes muestra dimensiones, tales como la consideración con los demás, el autocontrol en las relaciones sociales, el retraimiento social, la ansiedad social o timidez y el liderazgo.

\section{Relación entre acoso escolar y habilidades sociales}

A partir de las definiciones y aproximaciones conceptuales presentadas anteriormente, es pertinente mencionar la relación entre las dos variables. Para esto, Samper, Mestre, Malonda y Mesurado (2015) refieren que la aprobación o aceptación por parte de los compañeros del aula de clase es una concepción primordial para el desarrollo de las habilidades sociales, la socialización, la interiorización de valores y la capacidad de autorregulación emocional; además, genera fuente de apoyo emocional, seguridad y compañía en la persona. Según Estévez, Martínez y Jiménez (2009) los niños, niñas y adolescentes que se sienten reconocidos por sus iguales incrementan sus relaciones interpersonales y disponen de más recursos de apoyo, que a su vez contribuyen a un mayor bienestar y ajuste psicosocial. Además, un niño, niña o ado- 
lescente que se sienta valorado y aceptado, independientemente de su personalidad, características físicas o condición especial, dispone un espacio de interacción social positivo, determinado por el apoyo, la confianza y el respeto mutuo que se presente, mientras que los individuos que son victimizados perciben dicho contexto saturado de inseguridad y desconfianza, lo que conduce a un bajo rendimiento escolar (Samper et al., 2015).

De esta manera, un individuo que conoce y controla sus habilidades emocionales y sociales, que con facilidad puede interpretar los estados de ánimo de otras personas, es esa persona que puede operar en su entorno, de tal manera que su calidad de vida redunde positivamente (Contini, 2008). Así mismo, los niños, niñas y adolescentes que gozan de relaciones positivas con sus iguales, experimentan niveles altos de bienestar emocional, generando convicciones seguras de sí mismos, valorando las formas de conducta pro-social teniendo interacciones sociales más fuertes y adaptativas que quienes presentan carencia de ellas (Wentzel, Baker y Russell, 2009, citados en González, 2015).

De acuerdo con Salazar \& Narejo (2002) lograr resolver los conflictos presentados en el aula de clase de una manera positiva, demanda de un ciclo de formación e instrucción y en este caso, la educación en valores juega un papel importante. Además de esto, dentro de las conclusiones realizadas por los autores se dice que para luchar contra la violencia escolar es necesaria la participación activa de toda la comunidad educativa -incluyendo a padres de familia- en el desarrollo de actitudes de reflexión críticas sobre las problemáticas y conflictos que generalmente se presentan entre los estudiantes en el aula de clase.

Por otra parte, investigaciones recientes sobre victimización en el contexto escolar, según afirman Samper, et. al. (2015), han estado vinculados a los procesos cognitivos y emocionales como mecanismos y reguladores de control del comportamiento agresivo entre pares. Así las cosas, se considera que los puntos fuertes o deficientes para llegar a tener una adaptación social estable y fructífera en las relaciones sociales con los compañeros del aula de clase, están determinadas por las estrategias de afrontamiento y solución de problemas, las habilidades sociales y el auto-control que la persona logre obtener en la interacción con otros. Teniendo en cuenta, que las estrategias de afrontamiento que el niño, niña o adolescente desarrolle, pueden verse afectadas por el control excesivo de los padres, haciéndolas ineficaces ante las situaciones de conflicto y habilidades verbales y no verbales adecuadas y necesarias en el proceso educativo-social de la persona y más aún en procesos de victimización, los autores hacen hincapié en que en los programas de prevención e intervención sobre el acoso escolar deben trabajar en las relaciones del núcleo familiar.

De acuerdo con Frías, Fraijo y Cuamba, (2008) los padres de familia son núcleos importantes en la vida del niño, niña y adolescente en la medida en que contribuyen a facilitar a sus hijos la manera adecuada de conseguir y desarrollar habilidades sociales, emocionales y morales, para así prevenir que sean objeto de burlas, rechazo social, agresiones físicas, especialmente en lugares con poca supervisión de los adultos en el contexto educativo. Entre tanto, los autores afirman que el apoyo de los padres hacia sus hijos se relaciona con resultados positivos como la prevención de 
bajos niveles de ansiedad, depresión, disminución de conductas agresivas y alta autoestima, teniendo en cuenta que los docentes también juegan un papel importante en la generación adicional de información sobre los comportamientos que el menor está presentando en la Institución, esto con el fin de diseñar programas de detección temprana sobre un posible acoso escolar.

Por otra parte, Valadez (2008, citado en Uribe, Orcasita y Gómez, 2012) plantea que cuando se presentan interacciones a nivel personal con los demás seres humanos con los que se comparte un espacio, el uso y las normas de un territorio, son motivo de conflictos que pueden devenir en la violencia, entendida como agresión a otros. A partir de esto, el modelo que propone Bandura (1994, citado por Uribe, Orcasita y Gómez, 2012), para la prevención de dichos conflictos tanto en victimarios como en víctimas plantea los siguientes factores: desarrollo de habilidades sociales y de autocontrol, información, potenciación de la autoeficacia y apoyo social, debido a que al suscitar dichos factores se reafirman las modificaciones que los sujetos hacen en sus hábitos. Por tal razón, es importante considerar las implicaciones que tienen los factores propios del niño, niña y adolescente que de una u otra forma, favorecen el desarrollo y ejecución de conductas de riesgo para la salud física y/o psicológica, que terminan por perjudicar el desarrollo personal en términos de patologías y roles sociales. También, se deduce que los altos niveles de estrés y ansiedad tienden a afectar de manera negativa y progresiva las habilidades sociales de los individuos ya que conducen a que éste se perciba incapaz de mantener relaciones seguras, disminuyendo su bienestar y por ende el acceso al apoyo social.

A juicio de Rodríguez y Mejía (2012) las víctimas de acoso escolar son personas percibidas como débiles ante sus iguales, expuestos al desarrollo de conductas agresivas, tienen alteraciones del sueño y problemas en la alimentación, poseen pocas habilidades de comunicación y de socialización, baja autoestima, problemas para el manejo de la ansiedad, inseguridad y déficit en la toma de iniciativa. Por ende, a largo plazo el acoso escolar genera en las víctimas, dificultades para iniciar nuevas relaciones, sensaciones de aislamiento y pérdida de confianza, sintiéndose merecedores de lo que les está ocurriendo. Según Collel \& Escudé (2006) las víctimas clásicas son los niños y las niñas concebidos como más débiles que el resto de sus compañeros, que responden ante el agresor con llanto, dolor y escasas habilidades sociales (pocos amigos y sensibles a los rechazos).

Para Calderero, Caballo \& Carrillo (2011) las víctimas suelen mostrar déficit en habilidades sociales, autocontrol y estrategias interpersonales, características facilitadoras para la solución pacífica de los conflictos. Por todo ello, refieren que su intervención debe ser abordada en el aula de clases, debido a que es el contexto social donde se genera y se mantiene el acoso escolar, considerándose como un problema grupal, procedente de las malas relaciones que se generan entre los alumnos. Durante la intervención, mencionan que se realiza en primera instancia una evaluación de los comportamientos sociales, porque permiten la transferencia de las habilidades a otros contextos, permitiendo conocer no solo los déficits en habilidades sociales, sino también las fortalezas que posibiliten el aprendizaje de unas estrategias más adecuadas. (Caballo \& Monjas 2002; referidos en González, 2015). 


\section{Intervención en acoso escolar y habilidades sociales}

Dadas las preocupantes cifras de acoso escolar que se presentan, por ejemplo, en la ciudad de Bogotá, Colombia según Cepeda \& Caicedo (2012) en el sector de Ciudad Bolívar el 21.8 \% de los estudiantes refieren que la Institución Educativa se ha transformado en un lugar en el cual son maltratados de diversas formas: el 11.5\% considera que se hacen bromas crueles respecto a su aspecto físico, no hablan con ellos un $35.5 \%$, les dicen apodos que no les agradan un $26.1 \%$ y humillan y desprecian en público un 17. 8 \%. Así mismo en la Ciudad de Cali, Colombia en el estudio realizado por Paredes, Álvarez, Lega \& Vernon (2008) el 24.7 \% alcanza el porcentaje de agresores y un $24.3 \%$ el de víctimas, dejando entrever que una alta proporción de estudiantes están siendo víctimas y victimarios en los contextos educativos.

Según Musri (2012) en Paraguay el 38\% de los estudiantes son violentados por sus pares, de manera física o verbal y son pocas las Instituciones que tienen el respectivo conocimiento y toma de conciencia de la responsabilidad referente al fenómeno, ya que al parecer son muy escasas las que trabajan de una u otra forma en programas para prevenir el acoso escolar.

Además de esto, estudios realizados por Rigby (2003, citado por Cepeda \& Caicedo, 2012) evidencian que la propensión a victimizar a otros en el ámbito escolar predice la conducta antisocial y violenta de la persona adulta. De acuerdo con Maidel (2009) las personas agresoras demandan ayuda y orientación para el desarrollo eficaz de su autoestima, autoconfianza y la internalización de límites en su comportamiento.

Algunos programas están centrados en el mejoramiento de la convivencia escolar, como respuesta a la violencia implementando diferentes enfoques y líneas de actuación. Viedma (2007, citado por Gil, 2009) plantea que la convivencia es un fenómeno complejo, heterogéneo y multidimensional que se edifica a partir de las relaciones interpersonales que se fomentan en el diario vivir, el cual se reproduce en un escenario social delimitado y se ve alterado por un determinado contexto normativo y social. Así las cosas, diversas Instituciones Educativas sin necesidad de resolver conflictos o afrontar problemas de violencia, diseñan y ejecutan programas como mecanismo para mejorar la vida diaria de los estudiantes; otras Instituciones hacen hincapié en los docentes porque asumen que son más efectivos en generar bases sólidas y constructivas en la educación de los ciudadanos. Por último, Ortega (2009) refiere que, otras buscan articular las relaciones interpersonales al interior del aula, las relaciones entre los miembros de la Institución y entre las familias.

De este modo, para Gil (2009) dentro de los programas para la convivencia escolar se debe hacer la incorporación de estrategias de aproximación curricular que lleven consigo el desarrollo de estrategias de autocontrol de la agresión, de la ira y/o del estrés, de resolución de conflictos y desarrollo de habilidades de comunicación, entrenamiento asertivo, de empatía y de la autoestima. 
Un programa exitoso para el afrontamiento del acoso escolar debe tener en cuenta el fortalecimiento, desarrollo, entrenamiento en habilidades sociales para dotar a los niños, niñas y adolescentes de herramientas que les permitan interactuar de manera funcional con sus pares. Ballester \& Gil Llario (2002; citados en Lacunza, 2012) definen las intervenciones en habilidades sociales como un: "conjunto de estrategias y técnicas de la terapia de la conducta que tiene como finalidad la mejora en la actuación social de un individuo y su satisfacción en el ámbito de las relaciones interpersonales" (p, 37). Además de esto, los entrenamientos en habilidades sociales son experiencias de aprendizaje que radican en corregir conductas disfuncionales, observar un modelo de conductas adecuadas y practicar dichas conductas (García, Sanz \& Gil, 1998; referidos en Lacunza, 2012).

Es por esto que, Ferreira \& Muñoz (2011) proponen un programa de intervención en habilidades sociales para reducir el nivel de acoso escolar en las Instituciones Educativas ya que, éstas ayudan al individuo a tener las posibles herramientas específicas para poder integrarse al contexto social. Además, plantean que una de las grandes problemáticas que presentan tanto víctimas como agresores son las dificultades de habilidades sociales que permitan hallar soluciones asertivas a las situaciones de maltrato psicológico, físico y emocional. En este sentido, el programa llega a la conclusión a partir de sus resultados con estudiantes de quinto de primaria con edades comprendidas entre los 10 a 12 años -teniendo como variable independiente, el programa de intervención en el aprendizaje de habilidades sociales y utilizando el Instrumento Insebull (instrumento para la evaluación de Bullying)- que mediante un adecuado programa de intervención en habilidades sociales se pueden reducir los conflictos en el contexto escolar.

Collell \& Escudé (2006), afirman que una intervención centrada solamente en la víctima y/o agresor, genera efectos indeseables, culpabilizando a los protagonistas, a la vez liberando de toda imputabilidad a los demás estudiantes del grupo, dejando a un lado que son estos los que tienen capacidad para mantener o inhibir estas situaciones. Es por esto, que en la medida en que las estrategias educativas son decisivas, mediante el interés del alumno y de acuerdo al tipo de refuerzorecompensa, un programa de habilidades sociales debe tener en cuenta los siguientes objetivos (Álvarez, 1990; citado en González, 2015):

1. Desarrollo de habilidades de autonomía, ayudar, orientar y guiar al alumno a ser independiente.

2. Desarrollo de habilidades de interacción expresión de emociones, autoafirmación, saludar, hacer cumplidos y así mismo recibirlos de manera adecuada, comunicación verbal y no verbal entre otros posibles.

\section{Referencias}

Benítez, J. L., \& Justicia, F. (2006). El maltrato entre iguales: descripción y análisis del fenómeno. Revista de Investigación Psicoeducativa, 4(2), P.p. 81-93. Recuperado de http://www.investigacion-psicopedagogica.org/revista/articulos/9/espannol/Art_9_114.pdf 
Calderero, M., Caballo, V., y Carrillo, G. (2011). Acoso escolar y ansiedad social en niños (II): una propuesta de intervención en formato lúdico. Behavioral Psychology. 19(3), Pp. 611-626.

Clemence, A. (2001). Violence and security at school: the situation in Switzerland. En E. Debarbieux y C. Blaya (dirs.), Violence in schools. Ten approaches in Europe. Issy-les-Moulineaux: ESF editeur.

Caballo, V. (2007). Manual de evaluación y entrenamiento de las habilidades sociales. Madrid: Siglo XXI de España Editores S.A.

Campo, L. \& Martínez de Biava, Y. (2009). Habilidades sociales en estudiantes de psicología de una universidad privada de la Costa Caribe colombiana. Revista Iberoamericana de Psicología: Ciencia y Tecnología, 2(1), 39-51., Recuperado de: http://revistas.iberoamericana.edu.co/index.php/ripsicologia/article/ view/176/144

Castro, A. (2011). Violencia silenciosa en la escuela. Buenos Aires: Bonum

Cepeda, E., \& Caicedo, G. (2012). Acoso escolar: caracterización, consecuencias y Prevención. Universidad Nacional de Colombia. Pp. 5-13.

Cerezo, F. (2002). El bullying y su relación con las actitudes de socialización en una muestra de adolescentes. : Revista electrónica interuniversitaria de formación del profesorado, vol. 5 No. 1. P.p. 1-6 Recuperado de https://dialnet.unirioja.es/ servlet/articulo?codigo $=1031387$

Cowie, H. (1998). La ayuda entre iguales. Cuadernos de pedagogía, 270, 56-59.

Collell, J., \& Escudé, C. (2006). El acoso escolar: un enfoque psicopatológico. Anuario de psicología clínica y de la salud. (2), Pp. 9-14.

Contini, N. (2008). Las habilidades sociales en la adolescencia temprana: perspectivas desde la Psicología Positiva. Psicodebate, Psicología, cultura y sociedad, 9,P.p. 15-27. Recuperado de:http://www.palermo.edu/cienciassociales/ publicaciones/pdf/Psico9/9Psico\%2003.pdf

Congreso de Colombia, (2013). Ley No. 1620 del 15 de marzo de 2013 "por la cual se crea el Sistema Nacional de convivencia escolar y formación para el ejercicio de los derechos humanos, la educación para la sexualidad y la prevención y mitigación de la violencia escolar”. Recuperado de: http://wsp.presidencia.gov. co/Normativa/Leyes/Documents/2013/LEY\%201620\%20DEL\%2015\%20DE\%20 MARZO\%20DE\%202013.pdf

Currie, C., Gabhainn, S.N., Godeau, E., Roberts, C., Smith, R., Picket, W., Richter, M., Morgan, A., \& Barnekow, V. (2008). Inequalities in young people's healt. HBSC international report from the 2005/2006 survey. Recuperado de http:// www.euro.who.int/_data/assets/pdf_file/0005/53852/E91416.pdf

Del Prette, Z. \& Del Prette, A. (2008). Um sistema de categorías de habilidades sociais educativas. Paidéia, 18(41), 517-530. Recuperado de: http://www.scielo.br/ pdf/paideia/v18n41/v18n41a08.pdf 
Elliott, S., DiPerna, J., Mroch, A. \& Lang, S. (2004). Prevalence and patterns of academic enabling behaviors: An analysis of teachers' and students' ratings for a national sample of students. School Psychology Review, 33(2), 302-309. Recuperado de http://web.b.ebscohost.com/ehost/pdfviewer/pdfviewer?sid=12cd7c731d3f-46f3-be8c-f551f6009300\%40sessionmgr120\&vid=0\&hid=107

Enríquez, M. \& Garzón, F. (2015). El acoso escolar. Saber Ciencia y Libertad 10 (1), Pp. 219-233. Recuperado de file:///C:/Users/admin/Downloads/Dialnet-ElAcosoEscolar-5329121\%20(4).pdf P.p. 219-233

Estévez, E., Murgui, S. \& Musitu, G. (2008). Psychosocial adjustment in aggressors, pure victims and aggressive victims at school. European Journal of Education and Psychology, 1, Pp. 33-44. Recuperado de http://www.redalyc.org/ pdf/1293/129318696003.pdf

Estévez, E., Martínez, B. y Jiménez, T. I. (2009). Las relaciones sociales en la escuela: el problema del rechazo escolar. Psicología Educativa, 15(1), 5-12. Recuperado de: https://www.uv.es/lisis/belen/psicolog-educt.pdf

Frías, M., Fraijo, B., y Cuamba N. (2008). Problemas de conducta en niños víctimas de violencia familiar: reporte de profesores. Estudios de Psicología, Revista Scielo. 13 (1), Pp. 4-11.

Ferreira, Y. \& Muñoz, P. (2011). Programa de Intervención en Habilidades Sociales para reducir los niveles de acoso escolar entre pares o bullying. Ajayu, 9(2), 264283. Recuperado de http://www.scielo.org.bo/pdf/rap/v9n2/v9n2a4.pdf

Gairín, J., Armengol, C., \& Silva, B. (2013). El «Bullying» Escolar. Consideraciones Organizativas y Estrategias Para La Intervención. Facultad de Educación. UNED, Educación XX1. 16 (1), Pp. 17-38.

Garaigordobil, M., \& Oñederra, J. A. (2010). La violencia entre iguales. Madrid: Ediciones Pirámide.

García, A. (2010). Estudio sobre la asertividad y las habilidades sociales en el alumnado de Educación Social. XXI Revista de Educación, 12, P.p. 225-240. Recuperado de: http://rabida.uhu.es/dspace/bitstream/handle/10272/5352/b16450188. pdf

García Núñez del Arco, C. (2005). Habilidades sociales, clima social familiar y rendimiento académico en estudiantes universitarios. Liberabit Revista de Psicología, 11, 63-74. Recuperado de: http://pepsic.bvsalud.org/scielo.php?script=sci_art text\&pid=S1729-48272005000100008

Gil, J. (2009). Estrategias didácticas para mejorar la convivencia y participación del alumnado en educación física. Tesis Doctoral presentada al Departamento de Educación Fisica y Deportiva. Universidad de Granada, consultado en https:// hera.ugr.es/tesisugr/18585747.pdf

Goldstein, A. P., Sprafkin, R. P., Gershaw, N. L. y Klein, P. (1989). Habilidades sociales y autocontrol en la adolescencia. Barcelona: Martínez Roca. 
Gómez, S. (2015). Habilidades sociales de los escolares y prevención del conflicto: Programa de mejora del clima escolar. Tesis de grado presentado a la Universidad Autónoma Barcelona, consultado en https://ddd.uab.cat/pub/tfg/2015/133350/ TFG_sgomezserra.pdf

González, A. (2015). "Las Habilidades Sociales en los Fenómenos de Violencia y Acoso Escolar”. Escuela De Ciencia Sociales, Artes Y Humanidades. Pp. 1-65.

Kokkinos, C. M., \& Kipritsi, E. (2012). The relationship between bullying, victimization, trait emotional intelligence, self-efficacy and empathy among preadolescents. Social Psychological Education, 15, 42-58. doi: 10.1007/s11218-011-91689. Recuperado http://s3.amazonaws.com/academia.edu.documents/33157482/ KOKKINOS_KIPRITSI_2011_SOC_PSY_EDU.pdf?AWSAccessKeyId=AKI AIWOWYYGZ2Y53UL3A\&Expires=1488236307\&Signature=ylbXeeVYTqP O9aZlKpfGiWy\%2B3U8\%3D\&response-content-disposition=inline\%3B\%20filename\%3DThe_relationship_between_bullying_victim.pdf

Krug, E., Dahlberg, I., Mercy J., Zwi, A. \& Rafael, L. eds. (2002). World Report on Violence and Health. Recuperado de http://apps.who.int/iris/bitstream/10665/42495/1/9241545615_eng.pdf.

Lacunza, A., \& Contini, N. (2011). Las habilidades sociales en niños y adolescentes. Su importancia en la prevención de trastornos psicopatológicos. Fundamentos en humanidades. Vol XII, N²3, PP.- 159-182.

Lacunza, A. (2012). Las intervenciones en habilidades sociales: revisión y análisis desde una mirada salugénica. Psicología, Cultura y Sociedad. Pp. 63-84.

Lacunza, A., Caballero, S., \& Contini, E. (2012). Adaptación y evaluación de las propiedades psicométricas de la BAS-3 para población adolescente de Tucumán (Argentina). Revista Diversitas-Perspectivas en Psicología. Vol. 9, No. 1, P.p. 2944. Recuperado de: http://www.scielo.org.co/pdf/dpp/v9n1/v9n1a03.pdf

Lavilla, L. (2013). Las habilidades sociales en el marco de la orientación psicopedagógica. Revista de clases historia. Publicación digital de Historia y Ciencias Sociales. Artículo No. 365, P.p. 1-13

León, B. (2009). Salud mental en las aulas. Revista de estudios de Juventud, No. 84, P.p 63-84. Recuperado de http://www.injuve.es/sites/default/files/RJ84-06. $\mathrm{pdf}$

Maidel, S. (2009). "Cyberbullying: Um novo risco advindo das tecnologias digitais". Revista Electrónica de Investigación y Docencia. (REID), ISSN 1989-2446, Nº 2, págs. 113-119. Recuperado de: http://revistaselectronicas.ujaen.es/index.php/ reid/article/view/1158/980

Marín, A. \& Reidl, L. (2013). Validación psicométrica del cuestionario "así nos llevamos en la escuela" para evaluar el hostigamiento escolar (bullying) en primarias. Revista Mexicana de Investigación Educativa. Vol. 18 Núm 56 p 11-36. Recuperado de http://www.scielo.org.mx/pdf/rmie/v18n56/v18n56a2.pdf 
Martín, J. (2013). Aplicación de soci-escuela (Test Socio-métrico contra el acoso escolar) y Screening (para perfil psicológico de alumnos). II Curso de Verano. $\mathrm{N}^{\circ}$ 30, Pp. 10. Consultado en file://C:/Documents\%20and\%20Settings/practicanteseleccion/Escritorio/Dialnet-DirectricesYEstrategiasDeEvaluacionDeAlumnosConDis-4351699.pdf

Martínez-Monteagudo, M. C., Inglés, C. J., Trianes, M. V., \& García-Fernández, J. M. (2011). Perfiles de ansiedad escolar: diferencias en clima social y violencia entre iguales. Electronic Journal of Research in Educational Psychology, 9(25), 1023-1042. Recuperado de http://www.redalyc.org/pdf/2931/293122852003.pdf

Mavroveli, S., Petrides, K. V., Sangareau, Y., \& Furnham, A. (2009). Exploring the relationships between trait emotional intelligence and objective socio-emotional outcomes in childhood. British Journal of Educational Psychology 79, 259-272. doi: 10.1348/000709908X368848. Recuperado de: https://www.researchgate.net/profile/Adrian_Furnham2/publication/23413854_Relating_trait_ emotional_intelligence_to_objective_socioemotional_outcomes_in_childhood/ links/0c96053204adb77887000000/Relating-trait-emotional-intelligence-to-objective-socioemotional-outcomes-in-childhood.pdf

Michelson, L., Sugai, D. P., Wood, R. P. y Kazdin, A. E. (1987). Las habilidades sociales en la infancia: Evaluación y tratamiento. Barcelona. Martínez Roca

Monjas, M. (2004). Ni sumisas ni dominantes. Los estilos de relación interpersonal en la infancia y en la adolescencia. Memoria de Investigación, Plan Nacional de Investigación Científica, Desarrollo e Innovación Tecnológica. Valladolid, España. Recuperado de: http://cdd.emakumeak.org/ficheros/0000/0463/672.pdf

Musri, S. (2012). Acoso escolar y estrategias de prevención en educación escolar básica y nivel medio. Tesis presentada a la Facultad de Ciencias Humanas, de la Universidad Tecnológica Intercontinental, como requisito para la obtención del Título de Licenciado en Ciencias de la Educación. San Lorenzo - Paraguay, consultado en http://www.utic.edu.py/investigacion/attachments/article/61/ Tesis\%20Completa\%20SILVIA\%20MUSRI.pdf

Ortega, R. (2009). La convivencia escolar: qué es y cómo abordarla. Programa Educativo de Prevención del Maltrato entre compañeros y compañeras. Editorial Consejería de Educación y Ciencia. Junta de Andalucía.

Paredes, María., Álvarez, M., Lega, L. \& Vernon, A. (2008) "Estudio exploratorio sobre el fenómeno del "Bullying" en la ciudad de Cali, Colombia". Revista Latinoamericana de Ciencias Sociales, Niñez y juventud. 6(1): 295-317.

Polo, M., Barco, B., Castaño, E., Bullon, F., \& Carroza, T. (2015). Análisis de la Socialización sobre Perfiles de la dinámica bullying. Universitas Psychologica, 14(3), 1117-1128. http://dx.doi.org/10.11144/Javeriana.

Procuraduría General de la Nación. (2010). Ley No 1098 del 08 de Noviembre de 2006 "por la cual se crea el Código de Infancia y la Adolescencia". Recuperado de https://www.procuraduria.gov.co/portal/media/file/Visi\%C3\%B3n\%20Mundial_Codigo\%20de\%20Infancia\%202011(1).pdf 
Rodríguez, A., y Mejía Y. (2012). Bullying: un fenómeno por transformar. Duazary. Revista Dialnet. 9 (1), Pp, 98-104.

Salazar, M. y Narejo, N. (2002). Vías para abordar los conflictos en el aula. Revista electrónica interuniversitaria de formación del profesorado, 5, 1-4. Recuperado de: file://C:/Users/admin/Downloads/Dialnet-ViasParaAbordarLosConflictosEnElAula -1034377.pdf

Samper, P., Mestre, V., Malonda, E., y Mesurado B. (2015). Victimización en la escuela: relación de la crianza y variables funcionales-disfuncionales del desarrollo. Anales de psicología, Revista Scielo. 31 (3), Pp. 849-858.

Stan, C., Galea, I. (2014). The development of social and emotional skills of students - ways to reduce the frequency of bullying-type events. Experimental results Cristian Stan* Ioana Galea Beldean. Social and Behavioral Sciences. 114, Pp735-743.

Sánchez, C., \& Cerezo, F. (2011). Estatus Social de los sujetos implicados en bullying. Elementos para la mejora de la convivencia en el aula. Cuatrimestre. 22(2), Pp. 137-149.

Toledo, M. y Magendzo, A. (2011). Bullying: Avanzando hacia el pluralismo explicativo. Revista internacional Magisterio, No. 53, 16-22.

Uribe, A., Orcasita, L., Gomez, E. (2012). Bullying, redes de apoyo social y funcionamiento familiar en adolescentes de una institución educativa de Santander, Colombia. Psychologia: avances de la disciplina. 6(2), Pp. 83-99 